\title{
Implication of the Charge Site in "Charge-Remote" Fragmentations
}

\author{
Miquel Antoine and Jeanette Adams \\ Department of Chemistry, Emory University, Atlanta, GA, USA
}

() Am Soc Mass Spectrom 1992, 3, 776-778)

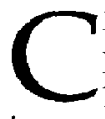

harge-remote fragmentations are a class of gasphase ion decompositions that have become important in the structural elucidation of many types of compounds [1]. Charge-remote fragmentations arise from closed-shell ions that contain a stable charge site. The decompositions are termed "chargeremoter $^{\prime \prime}$ because the reactions can be described by mechanisms that do not directly involve the charge site. For example, Gross and co-workers [2] originally proposed a mechanism for fragmentation of saturated fatty acid anions that involves a thermolytic-like, pericyclic, 1,4-elimination of $\mathrm{H}_{2}$ (Scheme I).

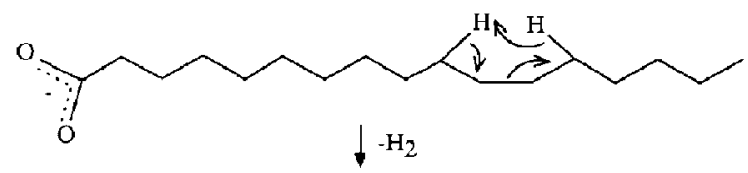<smiles>C=CCCCC</smiles>

Other charge-remote fragmentation mechanisms have since been proposed to describe decompositions of a large variety of compounds, including compounds that contain long alkyl chains [3-6] and others such as peptides $[7,8]$.

Questions have recently been raised concerning the "charge-remote" nature of these fragmentation reactions. Wysocki and Ross [5] suggested that the mechanisms might involve charge-mediated chemistry via ion-molecule complexes in which the charge-remote closed-shell ions result from loss of $\mathrm{H}$. from initially formed radical cations. Adams and coworkers [6], in their study of charge-remote allylic cleavages of deuterated monounsaturated fatty acid dęrivatives, showed some mechanistic inconsistencies that also suggested the possible intervention of the charge site. We have now further examined fragmentations of monounsaturated fatty acids by studying metastable

Address reprint requests to Jeanette Adams, Department of Chemistry, Emory University, Atlanta, GA 30322. ion and collision-induced decomposition of their [M + $2 \mathrm{Cat}-\mathrm{H}]^{+}$ions, in which Cat $=$alkali metal ion. These decompositions were studied previously $[9,10]$ by using mass-analyzed ion kinetic energy spectrometry. Unfortunately, the previously used energy scans provided poor product ion resolution so that some important fragmentation patterns were not discerned.

These fragmentation patterns, as studied by using first field-free region linked scans at a constant ratio $B / E$, involve formation of radical cations and specific changes in relative product ion abundances with increasing size of alkali metal ion. Examples of the chemistry are shown in Figure 1 for the $[\mathbf{M}+2$ Cat $\mathrm{H}^{+}$ions of cis-9-octadecenoic (oleic) acid, in which Cat $=\mathrm{Li}^{+}, \mathrm{Na}^{+}$, and $\mathrm{K}^{+}$. (Standard experimental procedures may be found in ref 4.) The spectrum of the $[\mathrm{M}+2 \mathrm{Li}-\mathrm{H}]^{+}$ions (Figure 1a) shows the classic $[6$, 10] pattern of charge-remote cleavages unique to monounsaturated fatty acid ions. Most product ions are closed-shell (odd-mass) ions that provide immediate information about the structure of the alkyl chain. Three of the four most abundant closed-shell ions involve cleavages in close proximity to the double bond (labeled $\mathbf{b}, \mathbf{c}$, and $\mathbf{d}$ ). There are a few open-shell (even-mass) radical cations, the most abundant being of $m / z 58\left(\mathrm{CO}_{2} \mathrm{Li}_{2}\right)^{+}$, which is labeled a, 72 $\left(\mathrm{C}_{2} \mathrm{H}_{2} \mathrm{O}_{2} \mathrm{Li}_{2}\right)^{\circ+}$, and $86\left(\mathrm{C}_{3} \mathrm{H}_{4} \mathrm{O}_{2} \mathrm{Li}_{2}\right)^{{ }^{++}}$. There is also a weakly abundant radical cation of $\mathrm{m} / \mathrm{z} 196$ (labelled c') that arises by cleavage of the allyl bond. Bambagiotti and co-workers [11] proposed that formation of the radical ion $c^{\prime}$ from fatty acid anions occurs via a charge-remote homolytic cleavage (Scheme II).

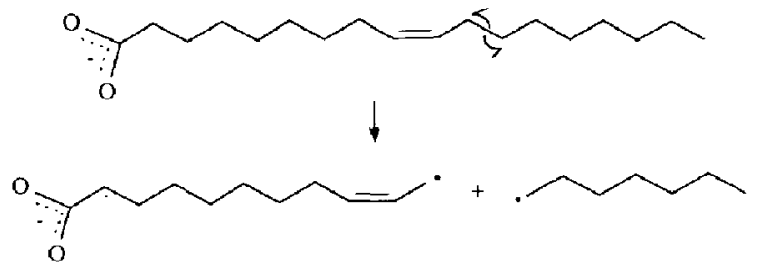

Others have since likewise argued in favor of this mechanism $[1,4,5]$. 

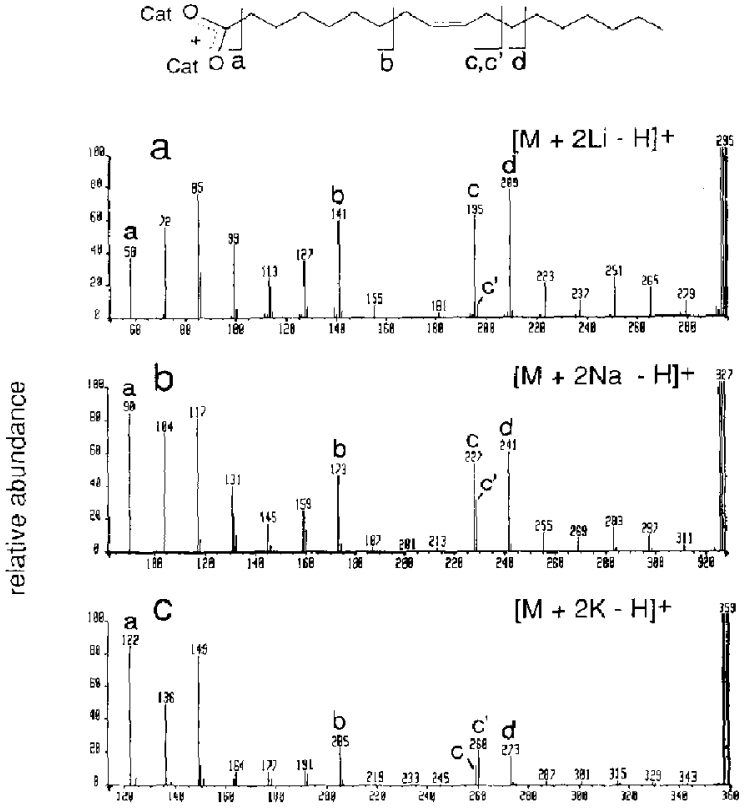

$\mathrm{m} / \mathrm{z}$

Figure 1. High-energy $(8-\mathrm{keV})$ collison-induced decomposition spectra of (a) $[\mathrm{M}+2 \mathrm{Li}-\mathrm{H}]^{+}$ions, (b) $[\mathrm{M}+2 \mathrm{Na}-\mathrm{H}]^{+}$ions and (c) $[\mathrm{M}+2 \mathrm{~K}-\mathrm{H}]^{+}$ions from cis-9-octadecenoic acid, desorbed by fast-atom bombardment and collided with $\mathrm{He}$ to reduce precursor ion beam transmission by about $70 \%$.

Comparing Figure 1a to $\mathrm{b}$ and $\mathrm{c}$ reveals some dramatic and surprising changes with increasing size of alkali metal ion. (Similar changes are also seen in spectra of $[\mathrm{M}+\mathrm{Cat}]^{+}$ions of monounsaturated fatty acids, alcohols, and esters.) The ones of particular interest here involve the relative abundances of the higher mass $c^{\prime}$ allylic radical cation $\left(\mathrm{C}_{11} \mathrm{H}_{18} \mathrm{O}_{2} \mathrm{Cat}_{2}\right)^{+}$ and the higher mass $\mathrm{c}$ allylic closed-shell ion $\left(\mathrm{C}_{11} \mathrm{H}_{17} \mathrm{O}_{2} \mathrm{Cat}_{2}\right)^{+}$. Effects of the size of the alkali metal ion on these two cleavages are summarized in Table 1 . Specifically, changing from $\mathrm{Li}^{+}$to $\mathrm{K}^{+}$causes a $450 \%$ increase in the $\mathbf{c}^{\prime}$ allylic radical cation, but the adjacent c allylic clnsed shell ion decreases by $64 \%$. In an attempt to elucidate the meaning of this chemistry, a series of experiments was conducted with increasing collision gas pressure. The results are provided in Table 2. For the $[\mathrm{M}+2 \mathrm{~K}-\mathrm{H}]^{+}$ions, the only fragmentations that occur at $0 \%$ beam reduction are for-

Table 1. Relative abundances of product ions from collision-induced decomposition of cis-9-octadecenoic acid cationized with $\mathrm{Li}^{+}, \mathrm{Na}^{+}$, and $\mathrm{K}^{+}$at $70 \%$ bearn reduction (see Figure 1)

\begin{tabular}{cccc} 
& \multicolumn{2}{c}{ Percentage of main beam } \\
\cline { 2 - 4 } Product ion & $\mathrm{Li}^{+}$ & $\mathrm{Na}^{+}$ & $\mathrm{K}^{+}$ \\
\hline$\left(\mathrm{C}_{11} \mathrm{H}_{17} \overline{\mathrm{O}}_{2} \mathrm{Cat}_{2}\right)^{+}$ & 0.36 & 0.35 & 0.13 \\
$\left(\mathrm{C}_{11} \mathrm{H}_{18} \mathrm{O}_{2} \mathrm{Cat}_{2}\right)^{+}$ & 0.04 & 0.20 & 0.22 \\
\hline
\end{tabular}

Table 2. Relative abundances of product ions from collision-induced decomposition of $[\mathrm{M}+2 \mathrm{~K}-\mathrm{H}]^{+}$ ions of cis-9-octadecenoic acid at different beatm reductions

\begin{tabular}{ccccc}
\hline Product ion & \multicolumn{3}{c}{ Percentage of main beam } \\
\hline & \multicolumn{3}{c}{ Percentage beam reduction } \\
\cline { 2 - 5 } & $0^{\mathrm{a}}$ & 10 & 30 & 60 \\
\cline { 2 - 5 }$\left(\mathrm{C}_{11} \mathrm{H}_{17} \mathrm{O}_{2} \mathrm{~K}_{2}\right)^{+}$ & 0.012 & 0.017 & 0.022 & 0.042 \\
$\left(\mathrm{C}_{11} \mathrm{H}_{18} \mathrm{O}_{2} \mathrm{~K}_{2}\right)^{++}$ & - & - & 0.012 & 0.051 \\
\hline
\end{tabular}

a It should be noted that $0 \%$ beam reduction does not imply metastable ion fragmentations because there is always residual fast-atom bombardment gas in the first field free region.

${ }^{b}$ The "__. symbols represent $<0.003 \%$

mation of the allylic closed-shell ion $c$ and the adjacent d closed-shell ion, of $m / z 259$ and 273, respectively, in Figure 1c, and loss of $K^{+}$[10]. The relative abundance of the $c^{\prime}$ radical cation is not significant until the beam is reduced by $30 \%$. (The charge-remote allylic fragment ion $b$, of $m / z 205$ in Figure $1 c$, also becomes abundant at this beam reduction.) At $60 \%$ beam reduction, many of the ions shown in Figure 1c are observed, but at slightly different abundances. Most importantly, however, the $\mathbf{c}^{\prime}$ radical cation, of $m / z 260$, becomes more abundant than the adjacent chargeremote fragment ion $\mathrm{c}$ of $m / z 259$.

This chemistry cannot be understood fully without further, more detailed studies. One fact is quite clear, however: the c closed-shell ions do not arise via a two-step radical mechanism from the adjacent $\mathbf{c}^{\prime}$ radical cations, as proposed previously [5]. If this were the case, the abundance of the $c^{\prime}$ allylic radical ion should decrease, and the abundance of the adjacent $c$ closedshell ion should increase, with increasing collision gas pressure. Such a trend is completely contrary to the data presented in Table 2. Formation of the $c$ closedshell ions is more likely via a rearrangement such as proposed by Adams and co-workers [6].

One other fact is also clear: The charge site must be affecting at least some of the "charge-remote" fragmentations, otherwise the two "charge-remote" fragment ion abundances would not be altered with changing size of metal ion. A simple argument that loss of $\mathrm{Cat}^{+}$is competing against some of the fragmentation routes cannot explain the data. Heterolytic loss of Cat ${ }^{+}$ increases with increasing size of $\mathrm{Cat}^{+}[10]$, and loss of $\mathrm{Cat}^{+}$from the linear structure shown in Figure 1 would compete best against the highest energy pathways. From Table 2, formation of the $c^{\prime}$ radical cation is a higher energy, faster route than formation of the $c$ closed-shell ion, and thus should compete less favorably with increasing size of Cat ${ }^{+}$. As shown in Figure 1 and Table 1, however, formation of $c^{\prime}$ out-competes formation of $\mathrm{c}$ with increasing size of metal ion, contrary to prediction.

The results in both Table 1 and Table 2 cannot be explained by simple kinetic arguments. The data can be understood, however, from competing decompositions of structurally distinct precursor ions. This idea is 

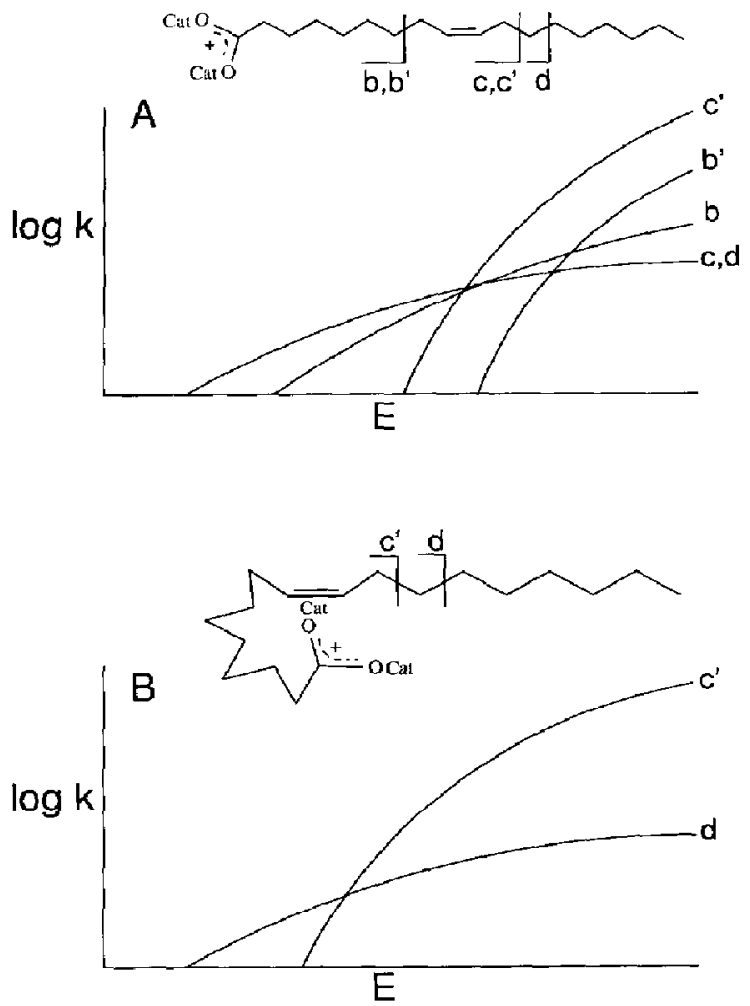

Figure 2. Schematic representations of $\log k(E)$ vs. $E$ curves that describe data in Figure 1 and Tables 1 and 2.

shown schematically in Figure 2. The linear structure shown in Figure $2 a$ would undergo classic chargeremote fragmentations via mechanisms previously proposed $[2,6,11]$, which include lower energy, slower rearrangements, and higher energy, faster homolytic direct cleavages $[1]$. In contrast, the cyclic structure shown in Figure $2 \mathrm{~b}$ in which the charge is stabilized by interactions with the double bond would undergo a charge-induced cleavage to give the allylic radical cation $c^{\prime}$ and perhaps other charge-remote cleavages such as d. As depicted in Figure $2 b$, the threshold energy for the charge-induced allylic cleavage would be significantly lower than that for the analogous charge-remote homolytic allylic cleavage in Figure 2a. Furthermore, the linear structure (Figure 2a) would give rise to a large number of product ions, whereas fragmentations of the cyclic structure (Figure $2 b$ ) would produce primarily $c^{\prime}$. (Tn produce $c, b$, and $h^{\prime}$ from the ryclic structure would require breaking the bond between $\mathrm{Cat}^{+}$and the double bond.) Thus, increasing the rela- tive population of the cyclic structure, even by a small amount, would cause the charge-induced radical cation $c^{\prime}$ to increase significantly. The probability of forming the cyclic structure would increase with increasing size of metal ion because the charge site would be increasingly destabilized in the linear structure but stabilized in the multi-bonded cyclic structure [12]. It appears that at high energies, fragmentation of the cyclic structure to give $\mathbf{c}^{\prime}$ is kinetically favored over isomerization to the linear structure, which would be then followed by fragmentation via the other routes.

We are continuing to evaluate this idea by studying formation of radical cations vs. closed-shell ions from other compounds, specifically in relation to changing the size of the metal ion. We also are studying other types of charged species, such as ones in which the charge site is sterically isolated from the double bond. We feel it would be premature for us to compare our data to previous findings regarding saturated ammonium ions [5] until we have also studied their chemistry. These results will be discussed in a future publication.

\section{Acknowledgment}

This research was supported by the Emory University Research Fund, the Petroleum Research Fund (25280$A C)$, which is administered by the American Chemical Society, and the National Science Foundation (CHE9113272). Support was also provided by an Emory Minority Graduate Fellowship. We also acknowledge M. T. Bowers for helpful comments.

\section{References}

1. Adams, J. Mass Spectrom. Rev. 1990, 9, 141-186.

2. Jensen, N. J.; Tomer, K. B.; Gross, M. L. I. Am. Chem. Soc. $1985,107,1863-1868$.

3. Adams, J.; Gross, M. L. J. Am. Chem. Soc. 1989, 111, 435-440.

4. Contado, M. J.; Adams, J. J. Anal. Chim. Acta 1991, 246, $187-197$.

5. Wysocki, V. H: Ross, M. M. Int. J. Mass Spectrom. Ion Proc. $1991,104,179-211$.

6. Contado, M. J.; Adams, J.; Jensen, N. J.; Gross, M. L. I. Am. Soc. Mass Spectrom., 1991, 2, 180-183.

7. Johtuson, R. S.; Martin, S. A.; Bieman, K.; Stults, J. T.; Watson, J. T. Anal. Chem. 1987, 59, 3621-3625.

8. Johnson, R. S.; Martin, S. A.; Biemann, K. Int. J. Mass Spectrom. Ion Proc. 1988, 86, 137-154.

9. Adams, J.; Gross, M. L. J. Am. Chem. Soc. 1986, 108, 6915-6921.

10. Adams, J.; Gross, M. L. Anal. Chem. 1987, 59, 1576-1582.

11. Bambagiotti A., M.; Corona, S. A.; Vincieri, F. F.; Petrucciani, T.; Traldi, P. Org. Mass Spectrom. 1986, 21, 485-488.

12. Hannongbua, S. V.; Rode, B. M. Inorg. Chem., 1985, 24, $2577-2580$. 